\title{
Uterine Transplant: Is this Non-vital Organ Transplant Worth the Effort?
}

Organ transplant is lifesaving in many different specialties of medicine. Uterine transplant not being lifesaving is only considered lifeenhancing, where a transplant is offered to women either born without it or having lost it, in a hope to achieve pregnancy and fulfill motherhood. With the possible option of gestational surrogacy, a surrogate can carry the biological child of the woman not having her own uterus or with uterine factor of infertility, following in vitro fertilization. However in some countries and religions, surrogacy and adoption is not permitted. The issue of risks and benefits of the option of uterine transplant and the surgical procedure it involves is debatable.

\section{History}

The animal trials began in dogs, pigs, rats and the first successful autotransplantation was performed in a dog as early as 1964 which was followed by delivery of a live offspring. This was soon accomplished by allotransplant of the uterus in a rat which was also successful with pregnancy and led to birth of a healthy offspring. ${ }^{1}$

The first reported human uterine transplant was carried out in Saudi Arabia in 2000; ${ }^{2}$ but was unsuccessful as it led to necrosis and loss of viability of the graft, which required its removal after 3 months. The second uterine transplant was performed by Ozkan et al. in Turkey in $2013 .{ }^{3}$ Although the uterus remained viable but there was only report of biochemical pregnancy. Brännström et al. ${ }^{4,5}$ initiated the first clinical trial of multiple transplantations, involving nine women who received uteri from live donors. After 6 months, seven uteri remained viable with regular menses and they reported the first successful uterine transplant that resulted in a live birth in a Swedish woman in 2014 following a transplant from her mother.

Till date, more than 15 babies have been delivered worldwide following uterine transplantation. Media flooded with news from the United Kingdom and United States of America reporting the first babies from uterine transplant. India too reported its first successful laparoscopic uterine transplant in Pune performed by Shailesh Puntambekar in 2017. ${ }^{6}$ This was also followed by live birth in 2018 . Recent publication appeared of a successful uterine transplant in a woman from a dead donor woman, this was followed by a pregnancy after seven months. ${ }^{7}$

\section{Donors}

For uterine transplantation, either live or deceased organ donor is acceptable with proven fertility. The World Health Organization recommends, organ donations from dead as this gives potential advantage of not risking live donors. Both live and dead donors have their advantages and disadvantages. When live donor is available, the surgery can be planned and scheduled; whereas in dead donors, it becomes emergency and sometimes may not be feasible, although the uterus can be removed with long vascular pedicles.

The surgery in the donor involves removal of the uterus with bilateral, long venous and arterial vascular pedicles. It includes extensive dissection of the pelvic sidewalls, which includes dissection of the ureters from their passages over the iliac vessel bifurcations distally to their inlets into the bladder, and dissection of the uterine veins and uterine arteries from their origin after separation of the ureter.

\section{Preparation of the Recipient}

The most important aspect behind the success of the uterine transplant is immunosuppression to prevent rejection of the transplanted uterus which involves use of corticosteroids and immunosuppressants like mycophenolate mofetil, thymoglobulin, anti-thymocyte globulin, tacrolimus and azathioprine.

The uterine transplantation is done by laparotomy, and with recent publication of laparoscopy, the surgical procedure becomes less invasive. The surgery involves synchronizing the donor and recipient procedures to have minimal cold ischemia time. Macrovascular technique which used part of aorta, vena cava, common and internal iliac vessels coupled with uterine arteriovenous tree enbloc with uterus for anastomosis was used by the Sweden study group. The transplantation also involves long surgical hours for the procedure to be accomplished. Pre-and postoperative thromboprophylaxis is essential component of surgery. Post-surgery, there is regular monitoring to detect early signs of rejection which involves clinical examination, doppler studies, cervical cultures and biopsies. Early signs of rejection have been detected on cervical biopsies, and the episodes were effectively reversed by short courses of increased immunosuppression. ${ }^{4,5}$

\section{Pregnancy after Uterine Transplant}

The reported pregnancies were following in vitro fertilization and it is imperative to continue immunosuppressive therapy throughout pregnancy. The three aspects of pregnancy which need mention are the effect of pregnancy on graft and vice versa, the effect of immunosuppressants on fertility and pregnancy outcome. The most important aspect of uterine transplant was its ability to function, maintain strength and perform its function in response to growing fetus and the hormonal and metabolic functions.

In 2014, Brannstrom and his team had reported the birth of first baby following uterine transplant in a woman who had a uterine transplant from her own mother. She started menstruating from day 43 following surgery and conceived following in vitro fertilization after 12 months of her transplant. She received immunosuppression with corticosteroids, tacrolimus and azathioprine. She developed preeclampsia and had her cesarean at 31 weeks to deliver a healthy baby boy. ${ }^{4}$

In the other four pregnancies of the Swedish trial, the recipients showed resolvable episodes of mild rejection and the children were born healthy. ${ }^{5}$ 


\section{Risks and Complications of Uterine Transplantation}

The most important aspect of uterine transplant is to understand as a surgeon and counsel the woman that it is a complex procedure and it involves major surgical procedure and associated complications. The other problems are graft rejection and sepsis associated. The use of immunosuppressants and the health implications need addressal and consent. The easier option is to have a child through surrogacy. The woman or the couple should give consent after understanding that even life risk is involved and an unpublished case of death in a transgender woman is reported. Graft rejection is reported in best of the centers. In the developing countries such surgical benefits should be weighed against the involved cost and probably should involve ethical clearance and auditing to regulate performing uterine transplants in most optimal research settings.

\section{CONCLUSION}

The research and skill development in performing uterine transplant is expected to advance as the desire to carry a baby in a woman in her own womb may overpower the risks involved in this procedure. New surgical techniques are expected to evolve in near future and may attain importance. Presently, it is not answerable whether this will stand the test of time.

\section{Shalini Gainder Additional Professor Department of Obstetrics and Gynecology PGIMER, Chandigarh, India}

\section{REFERENCES}

1. Johannesson L, Enskog A, Molne J, et al. Preclinical report on allogeneic uterus transplantation in non-human primates. Hum Reprod. 2013;28(1):189_ 198.

2. Fageeh W, Raffa H, et al. Transplantation of the human uterus. Int J Gynaecol Obstet. 2002;76(3):245-251.

3. Ozkan O, Akar ME, Ozkan O, et al. Preliminary results of the first human uterus transplantation from a multiorgan donor. Fertil Steril. 2013;99(2):470-476

4. Brannstrom M, Johannesson L, et al. Livebirth after uterus transplantation. Lancet. 2015;385(9968):607-616.

5. Brannstrom M. The Swedish uterus transplantation project: the story behind the Swedish uterus transplantation project. Acta Obstet Gynecol Scand. 2015;94(7):675-679.

6. Puntambekar S, Telang M, et al. Laparoscopic-assisted Uterus Retrieval from Live Organ Donors for Uterine Transplant. J Minim Invasive Gynecol. 2018;25(4):571-572.

7. Ejzenberg $D$, Andraus $W$, et al. Livebirth after uterus transplantation from a deceased donor in a recipient with uterine infertility. The Lancet 2018;392(10165): P2697-2704. 